\title{
Galaxy Collisions, Gas Stripping and Star Formation in the Evolution of Galaxies
}

\author{
Jan Palouš \\ Astronomical Institute, Academy of Sciences of the Czech Republic \\ Boční II 1401, 14131 Praha 4, Czech Republic \\ palous@ig.cas.cz
}

\begin{abstract}
A review of gravitational and hydrodynamical processes during formation of clusters and evolution of galaxies is given. Early, at the advent of $N$-body computer simulations, the importance of tidal fields in galaxy encounters has been recognized. Orbits are crowded due to tides along spiral arms, where the star formation is enhanced. Low relative velocity encounters lead to galaxy mergers. The central dominating galaxies in future clusters form before the clusters in a merging process in galaxy groups. Galaxy clusters are composed in a hierarchical scenario due to relaxation processes between galaxies and galaxy groups. As soon as the overall cluster gravitational potential is built, high speed galaxy versus galaxy encounters start to play a role. These harassment events gradually thicken and shorten spiral galaxy disks leading to the formation of SO galaxies and ellipticals. Another aspect of the high speed motion in the hot and diluted Intracluster Medium (ICM) is the ram pressure exerted on the Interstellar Matter (ISM) leading to stripping of the ISM from parent spirals. The combinations of tides and ram pressure stripping efficiently removes the gas from spirals, quenching the star formation in galactic disks, while triggering it in the tidal arms and at the leading edge of gaseous disk. Gas stripping from disks transports the metals to the ICM. In some cases, the gas extracted from the galactic disks becomes self-gravitating forming tidal dwarf galaxies.

Star formation (SF) is another important ingredient in the evolution of galaxies. Young stars provide the energy, mass feedback and the metals to the ISM. SF also drives the supersonic turbulence and triggers SF at other places in the galaxy. Examples of supershells in the ISM, resultant from the evolution of stellar cluster, are given. Structures, supershells, filaments and sheets are also produced when the ISM is compressed during galaxy collisions and when high velocity clouds (HVC) fall and ram through gaseous galaxy disks. In some cases, the compressed structures become self-gravitating and fragment to produce clumps. When a low density medium is compressed, the clump masses and sizes correspond to those of giant molecular clouds (GMC). When already existing GMC's are compressed they are driven to collapse forming massive super-star clusters. The fragmentation process in the dense environment of GMC's produces fragments of stellar masses with the mass function having a slope similar to the stellar Initial Mass Function.
\end{abstract}




\section{Introduction}

The internal evolution of isolated galaxies is the subject of many studies. The evolution of stellar disks depends on the ratio between the radial component of the velocity dispersion $\sigma_{R}$ multiplied with the local epicyclic frequency $\kappa$, which is an analog of pressure in the stellar disk, and the disk surface density $\Sigma$ multiplied with the constant of gravity $G$, which gives the local gravitational force in the flat disk. The above quantities are combined in the Toomre (1964, 1977) $Q$ parameter

$$
Q=\frac{\sigma_{R} \kappa}{3.36 G \Sigma}
$$

to evaluate the local stability of a rotating, self-gravitating disks: for $Q<1$ the disk is locally unstable and forms large scale deviations from the axial symmetry - spiral arms. A purely stellar disk heats up, when individual stars scatter on spiral arms, and self-stabilizes.

If the fraction of the disk kinetic energy in random motions is small enough, the bar forms in its central part (Ostriker \& Peebles, 1973). The bar exchanges

the angular momentum with stars, which results in radial redistribution of mass leading to the formation of a central mass concentration. The growing central mass concentration partially dissolves the bar itself (Shen \& Sellwood, 2004).

A dissipative gaseous interstellar medium (ISM) is an additional component of the disk moving in a common gravitational field with stars. Energy dissipation, supersonic shocks and collisions of interstellar clouds reduce random motions of the ISM agitating spiral-like instabilities, which gradually heat the stellar disk. In an isolated spiral galaxy, the heating by a bar and spiral arms increases the stellar velocity dispersion by less than a factor of 2 (Bournaud et al., 2004).

The ISM complements the influence of the central mass concentration with viscous forces that shift systematically the gas flow-lines relative to the stellar orbits. The evolution of a bar and spiral structure in an isolated galaxy, their growth and dissolution, is driven by a combination of the increase in stellar velocity dispersion due to scattering of individual stars on large scale deviations from the axial symmetry, by the growth of the central mass concentration and by the torque between the gaseous and stellar components (Bournaud \& Combes, 2004).

Star formation, the mass recycling between stars and ISM, and the energy feedback are also recognized as fundamental processes. They are included in closed box models the evolution of isolated galaxies (Jungwiert et al. 2001, 2004). Several issues are addressed, remaining as open question if, when, and how much the box has to be open to gas infall, environmental effects, and galaxy major and minor mergers:

- Persistence of star formation. With the present rate, star formation would consume all the gas within less than a few Gyr. Fresh gas supplies are needed to keep the star formation active. 
- The metalicity distribution and chemical evolution of the disk. The G-dwarf problem requires gas infall.

- The existence of a thin gaseous disk, where recurrently the instabilities operate also needs accreted fresh gas.

- Thin disks are destroyed in minor merger and harrasment events. They have to be recreated.

- Renewal of bars driving the mass to the galactic center also requires gas accretion.

In this review, the tides, major, intermediate and minor mergers events, harrasment events, gas stripping, star formation and feedback are described. We discuss the relevance of these processes in the formation, and evolution of galaxies, and in the evolution of galaxy groups and clusters.

\section{Tides}

The importance of tides has been shown in early numerical N-body simulations by Toomre \& Toomre (1972). Their restricted N-body simulations of an encounter between two spiral galaxies led to the basic features. As the galaxies pass by each other, tidal forces provide the stars and ISM of the disks with sufficient energy to escape the inner potential well. Two streams of disk material appear on both near and far side of the disks relative to the position of the peri-center. In the near side, stars and gas form a tidal bridge between the disks while the far side material forms long tidal tails. The length and the shape of the tails is a sensitive function of the relative velocity and of the geometry of the encounter: the most effective are the low relative velocity prograde encounters, where the spin and angular momentum vectors are aligned. In this case, the momentum and energy exchange in resonant orbits is most effective, leading to long tidal arms and a close interconnection between the central parts of the original disks, to merge in the future.

The evolution of tidal debris is described by Mihos (2004). Most of the remnant material remains bound. It follows elliptical orbits and only a small fraction of the orbits are unbound. The fraction of gravitationally bound material is proportional to the extend of the dark matter halo: large halos result in less unbound material. The bound tidal debris return after some time to the galaxy disk in the form of gas infall and high velocity clouds, which may trigger the star formation. A fraction of the disk gas on unbound orbits becomes part of the Intracluster medium (ICM). The metalicity of the ICM, which is about $1 / 4 Z_{\odot}$, gives an evidence of the connection between galaxy disks and ICM. The slow encounters are the most effective in driving the gas of the original disks inward to the center of the future galaxy produced by the merger, which triggers a nuclear starburst. 


\section{$3 \quad$ Major mergers}

Computer simulations of low velocity encounters of spiral galaxies with similar masses - major merger events - produce remnants that are in surprisingly good agreement with the observed shapes, density profiles and velocity distribution of the observed giant elliptical galaxies. The mass ratio of the progenitor disks determines the global properties of the remnant (Burkert \& Naab, 2003a, b). Giant elliptical galaxies can be subdivided into disky and boxy types (Naab \& Burkert, 2003): the less massive disky giant ellipticals show diskto-bulge rations of $\mathrm{S} 0$ galaxies and rotation in their outer parts. They can be understood as resulting from major mergers with mass ratios from $1 / 1$ to $1 / 4$. The more massive boxy giant ellipticals form by $1: 1$ mergers with special initial orientations only, which weakens the disk merger scenario as the possible formation mechanism. Other processes have to be added, such as the star formation and the energy dissipation in the gaseous components of progenitor disks.

The present day giant elliptical galaxies may have been formed by major mergers of gas-rich galaxies with a subsequent starburst, or by mergers of less gas-rich galaxies, which is more common in galaxy clusters. The second scenario assumes that a fraction of stars forms before ellipticals in smaller gasrich galaxies. In the $\Lambda \mathrm{CDM}$ cosmological simulations the number of mergers varies with $\mathrm{z}$. The giant haloes of future elliptical exist already at $z=6$ with the relatively small variation of mass at $z<6$. Giant ellipticals exist at redshifts $z=3$ with about $50 \%$ of stellar mass, however, a typical $\mathrm{cD}$ galaxy has suffered significant merging events even at redshifts $z<1$ (Gao et al., 2004). Bimodal distribution of metalicity observed in elliptical galaxies is a product of gas-rich mergers, when the globular clusters form in the tidal arms (Li at al. 2004).

The Antennae, the colliding pair of galaxies NGC 4038/39, provide an nearby example of an early phase of a low velocity encounter between galaxies. The velocity field of Antennae galaxies, showing the details of the interaction, has been measured by Amram et al. (1992). Later, when the collision partners will not be distinguished any more, it should result in a field elliptical galaxy. Another example of a slightly more advanced ongoing merger event, is the Hickson compact group 31 (Amram et al. 2004). HCG 31 is a group in early phase of merger growing through slow and continuous acquisition of galaxies from the associated environment. One of the best examples of remnants after a recent merger event is the giant elliptical galaxy NGC 5018. Its inner part shows a uniform $\sim 3$ Gyr old stellar population presumably produced in a merger induced starburst (Buson et al., 2004).

\section{Intermediate and minor mergers}

Collisions of intermediate $(1 / 5-1 / 10)$ mass ratio partners show the intermediate merger events, which are explored by Bournaud et al. (2004). They 
result in a hybrid system with spiral-like morphology and elliptical-like galaxy kinematics similar to some of the objects identified by Chitre \& Jog (2002).

Minor mergers, i.e. merger of a galaxy with a satellite $1 / 10$ or less massive than the galaxy itself, are discussed in the context of formation of thick disk and of disk globular clusters (Bekki \& Chiba, 2002, Bertschik \& Burkert, 2003). The disk globular clusters are formed in the high-pressure dense central region of the gas-rich dwarf galaxy, which is compressed in a tidal interaction with the thin disk of the spiral galaxy, and later they are dispersed in the disk region, when the original dwarf merges with the galaxy. The origin of thick galactic disks may be the result of the same minor merger event producing the disk globular clusters.

\section{Evolution of galaxies in clusters}

An overabundance of spiral galaxies and an under abundance of S0 galaxies in high-redshift clusters, when it is compared to low $z$ clusters, is a consequences of galaxy merger events and of other environmental effects. In $\Lambda$ DCM cosmological models with $\Omega_{0}=0.4$ and $\Omega_{\Lambda}=0.6$, the galaxy interactions and tidal effects play a rôle mainly at intermediate redshifts $0.5<z<5$ (Gnedin, 2003). Galaxies entering clusters with low relative velocities merge their halos with the cluster and their subsequent dynamical evolution is due to perturbations along their orbit. The infalling galaxy groups experience merger events of their members. After virialization, when the galaxy velocity in the cluster increases to a few $10^{3} \mathrm{~km} \mathrm{~s}^{-1}$ the mergers are suppressed and high-speed galaxy encounters and interactions with the intracluster medium (ICM) start play a role.

The ICM consists of hot $\left(\sim 10^{7} \mathrm{~K}\right)$ diluted $\left(10^{-3}-10^{-4} \mathrm{~cm}^{-3}\right)$ gas, which is detected in X-ray observations. The optical/infrared observations (Renzini, 1997) monitor the stellar component of galaxies, and also their chemical composition. The bulk of the cluster light is produced in bright giant elliptical galaxies and in galactic bulges by intermediate and low mass stars. The content of Fe in clusters scales with their total light and the abundances of various elements does not seems to change from cluster to cluster (Renzini, 2004). If the metals are mainly produced in SN Type Ia and SN Type II, the constancy of metallic ratios implies the same universal ration of the two types of SN. It may be interpreted as a sign that majority of cluster metals have been produced in the dominating giant galaxies, and that the stars formation holds a universal initial mass function (IMF). It appears that the total mass of metals in the ICM supersedes the total mass of metals in stars (Renzini, 2004). The galaxies lose more metals to the ICM than they are able to retain. Their total abundance does not correspond to the actual SN rates, the observations could be reproduced if the SN Type Ia in ellipticals have 5 - 10 time larger rates in the past compared to present and if the slope of the IMF is not too shallow: Salpeter (1955) IMF with the power law slope -2.35 matches the requirements. 
How the metals were transported to the ICM? Potential mechanisms are the ICM ram pressure stripping of galactic ISMs, which may become more effective in combination with gravitational tides, or the star formation feedback connected to early stellar winds driven by the starburst forming majority of the stellar galaxy itself. An evidence for early stellar winds serve the Lymanbreak galaxies (Pettini et al., 2003). Another possibility are late local winds due to massive starbursts (Heckmann, 2003) or flows driven by a declining rate of SN type Ia (Ciotti et al., 1991). Both, gas extraction by stripping and gas ejection by winds will be described below.

\section{Galaxy harrasment}

Moore et al. (1996) discuss the heating effects influencing sizes and profiles of dark matter haloes of galaxies in clusters: tidal heating of haloes on eccentric orbits and impulsive heating from rapid encounters between haloes. N-body simulations show that both processes restrict the halo sizes. Frequent high speed galaxy encounters in clusters, galaxy harassment, drive the morphological transformation of galaxies in clusters. Moore et al. (1995) show the dramatic evolution, which happened in clusters during the recent $\sim 5$ Gyr. Both, young clusters at $z \sim 0.4$ and old nearby clusters have central dominant elliptical galaxies, which have been formed before clusters. The young clusters are populated by many spiral galaxies, but the old, large virialised systems, have inside of a deeper potential valley all other galaxy types, like S0, dwarf Ellipticals, dwarf S0, dwarf Spheroidals and Ultra Compact Dwarfs (Moore, 2003). Numerical simulations show that if the cluster velocity dispersion is more than a few times the internal velocity dispersion of galaxies, they do not merge in encounters. The observed morphological change is due to impulsive interactions during high speed encounters restricting the dark matter haloes and pumping the kinetic energy into disks of spirals changing them to So, dE, dS0, sSph, UCD galaxies. Hydrodynamical processes, like gas stripping, also matters, and help with the change, particularly in relation to star-formation and ICM.

\section{$7 \quad$ Gas stripping}

Gunn \& Gott (1972) in a description of events during and after the collapse, or formation of a galaxy cluster, predict the formation of hot and diluted intracluster medium (ICM) produced in shock randomization of infalling gaseous debris. The interstellar medium (ISM) in a galaxy moving through the ICM feels the ram pressure $P_{r}$, which is proportional to

$$
P_{r} \sim \rho_{I C M} v^{2},
$$

where $\rho_{I C M}$ is the density of the ICM and $v$ is the velocity of the galaxy relative to the ICM. The ISM may be stripped away the parent galaxy if at given 
distance from the galactic center the ram pressure exceeds the gravitational restoring force $F_{r}$

$$
F_{r}=2 \pi G \Sigma_{s} \Sigma_{I S M}
$$

where $\Sigma_{s}$ is the surface density of stars, $\Sigma_{I S M}$ is the surface density of the ISM and $G$ is the constant of gravity.

The ram pressure gas stripping influences the presence of the ISM, which may affect the star formation rates in galaxies. It is still an open question, if the observation of Butcher \& Oemler (1978, 1984), showing that distant clusters contain a far higher fraction of blue star forming galaxies than their near-by counter-parts, can be explained with the ICM ram pressure stripping of the ISM from galaxies.

A mechanism that suppresses the star formation in local galaxy clusters is apparently connected to the morphological transformation of galaxies: S0 galaxies are under-represented in distant galaxy clusters, luminous spiral galaxies are in deficit near the centers of local galaxy clusters (Dressler et al., 1997; Couch et al. 1998). This suggests that the cluster environment removes the ISM from galaxies, suppressing star formation and transforming spirals to S0's.

Numerical simulations of ram pressure gas stripping using 3-dimensional SPH/N-body code have been preformed by Abadi et al. (1999). They confirm the predictions of Gunn \& Gott (1972) that the radius to which the gas is removed from the parent galaxy depends on the relation of the ram pressure to the restoring force. But in any case a substantial part of the cold gas remains sufficiently bound to the stellar disk. The star formation rate is reduced by a factor of 2 only, which brings them to a conclusion that the simple ram pressure stripping does not adequately explain the sharp decline of star formation seen in Butcher-Oemler effect.

Volmer et al. (2001) show in simulations using sticky particles that the gas stripping is rather sensitive to the galaxy orbit, particularly to the minimum distance to the cluster center, and also to the orientation of the galactic disk relative to the orbit inside the cluster. In some cases the removed ISM is re-accreted and it falls back to the galactic disk, possibly triggering star formation in the central part of the disk within the remaining gas. Simulations of ram pressure gas stripping along an orbit in a cluster by Jáchym (2004) give similar result: the orbital parameters and the orientation of the galaxy are important. The gas is more effectively removed when the pole of the galactic plane is near the direction of the galaxy orbital motion in a cluster (Fig. 1) compared to the situation when the galaxy moves edge-on along its orbit.

\section{Gas stripping and tides}

Recently reviews on the efficiency of the stripping mechanism were given by van Gorkom (2004) and Combes (2004). The theoretical considerations stress the effects of viscosity and thermal conduction including Kelvin-Helmholtz instability (Nulsen, 1982). The turbulence, shells and supershells formed by 

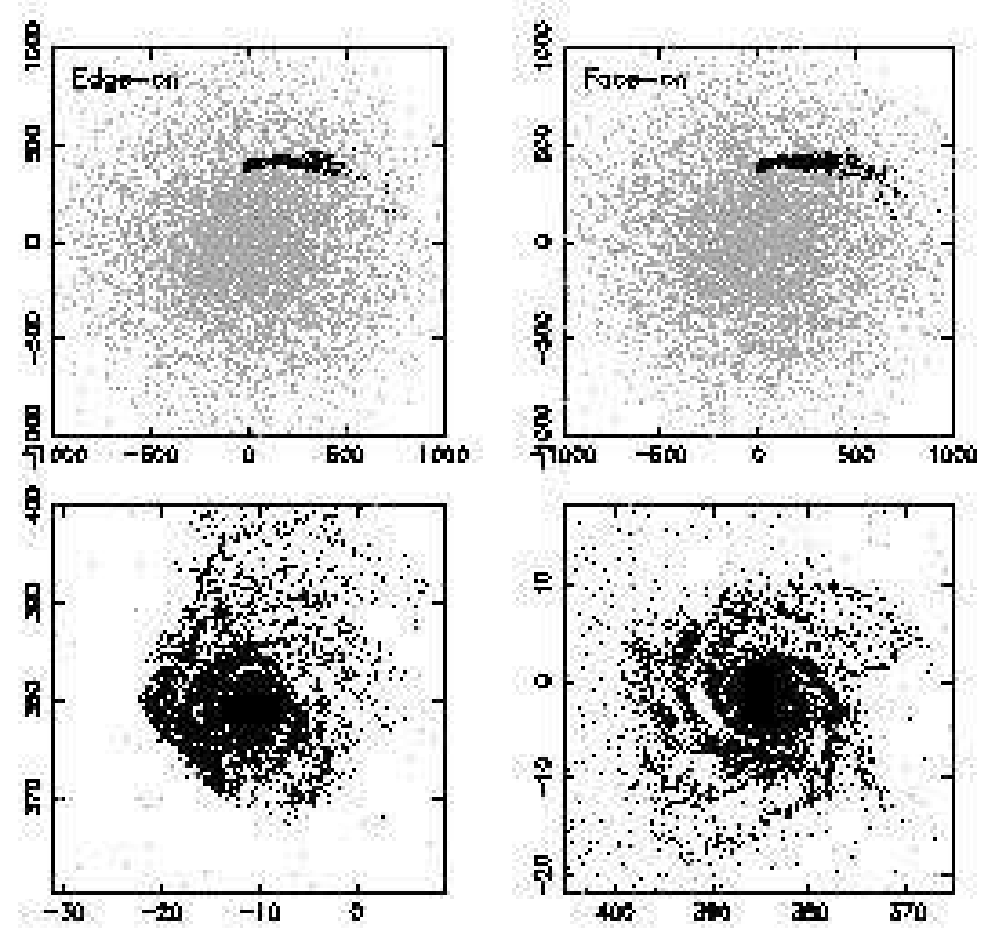

Figure 1: Simulations of ram pressure gas stripping (Jáchym, 2004). Edge-on orientation of the galaxy plane relative to the orbit (left panel) is compared to the pole-on orientation of the galaxy plane (right panel).

star formation increase the effect of the ram pressure stripping due to erosion at the edges of ISM holes (Bureau \& Carignan, 2002). Ram pressure also contributes to the overpressure inside of expanding structures increasing their sizes.

A proto-type of overwhelming gas stripping in the Virgo cluster is the galaxy NGC 4522 with a truncated HI disk, enhanced star formation in the central region, extraplanar gas, while undisturbed stellar disk. The gas distribution suggests an ICM-ISM interaction, while the undisturbed disk rules out a gravitational interaction (Kenney \& Koopmann, 1999).

The transformation of galaxies in dense environment results from the combined action of gas stripping and gravitational tides. C 153 galaxy in the cluster Abel 2125 shows an ongoing gas stripping: A tail of ionized gas is seen in [O II] emission, which extends at least $70 \mathrm{kpc}$ toward the cluster core along C 153 orbit, coincide with a soft X-ray feature seen in the Chandra observations (Keel et al., 2004). At the same time the HST optical picture shows clumpy morphology, including luminous star-forming complexes and chaotic 
dust features. The perturbed stellar disk with enhanced star formation activity suggests a possibility that a burst of star formation has been initiated during the close passage of $\mathrm{C} 153$ to the cluster center.

More examples of the stripped dwarf irregular galaxies (van Zee et al., 2004) and of galaxies with truncated star formation disks in Virgo cluster (Koopmann \& Kenney, 2004a, b) show both the gas stripping and gravitational interactions including the induced star formation, however, the dominant environmental effect on galaxies in clusters is the ram pressure gas stripping.

\section{Tidal Dwarf Galaxies}

The long tidal tails observed in many cases of interacting galaxies show massive clumps of $10^{9} M_{\odot}$ (e.g. IC 1182, NGC 3561, NGC 4676, etc.). These massive blobs has been named Tidal Dwarf Galaxies (TDG), since they have galactic masses and the chemical composition corresponds to the recycled matter pushed out of the disks of the interacting partners. To become a long living independent galaxy they should be gravitationally bound systems (Duc et al., 2000). The most prominent interacting system, the Antennae galaxies, has been studied by Mirabel et al. (1992), who describe a TDG candidate at the tip of their long tidal arm. Hibbard et al. (2001) also analyzed this concentration of gas and star forming regions, however, from observations it is very difficult to assess if it is gravitationally bound.

To decide if a TDG candidate will be a new galaxy formed out of an interaction, several questions have to be addressed (Bournaud et al. 2004):

- Are the blobs real concentrations in three dimensions? They may be just projection effects due to tidal arm geometry.

- Are they kinematically decoupled from the tidal arms? Are they long living?

- Do they contain dark matter?

Bournaud et al. (2004) give the answer at least to the first question: The simulations of galaxy collisions provide shapes of tidal arms, which may be virtually observed from all the sides. They conclude that some observed $10^{9} M_{\odot}$ mass concentrations are real TDG candidates. Some of them are selfgravitating, but to decide on their future is still difficult with the current resolution of simulations. The star formation, energy and mass feedback have to be included in the future numerical experiments. The third problems on the content of the dark matter also remains open due to the uncertainty in the internal kinematics of TDG candidates.

The simulations provide one more important conclusion (Duc et al. 2004): the existence of $10^{9} M_{\odot}$ mass concentrations at the tips of tidal arms is rather sensitive to the extent and the density profile of the halo. It has to be extended enough ( $\sim 150 \mathrm{kpc}$ from the center of a collision partner) so that the 
collision happens within it. Then the flow lines of the perturbed gas from all galactocentric distances in the original disk concentrate at the tips of the tidal arms, giving a kinematical origin to the TDG. When the halo is too concentrated so that the collision happens at the distance, where the rotation curve already decreases, the perturbed gas populates all the tidal arm and there is no place where $10^{9} M_{\odot}$ may gather.

The kinematical gathering of stars and gas distinguishes TDG from superstar clusters (SSC), which are seen the interacting galaxies. SSC are not only less massive $\left(10^{5}-10^{7} M_{\odot}\right)$ but they arise from gravitational instabilities in the stellar or gaseous components along the tidal arms.

\section{SMC, LMC and the Milky Way system}

The Small (SMC), and Large Magellanic clouds (LMC) and the Milky Way form the nearest interacting system of galaxies, where the gravitational and hydrodynamical processes can be studied. We see the result of a combination of gravitational tidal forces with gaseous ram pressure. The star formation and the mass and energy feedback is also involved as demonstrated with many expanding ISM shells and supershells.

The high resolution HI surveys of the LMC (Kim et al. 1998, 1999), of the SMC (Staveley-Smith et al. 1997, Stanimirovich et al. 1999), of the Magellanic bridge region (Muller et al. 2003) and of all the system (Büns et al. 2004) have been preformed with the Australia Telescope Compact Array and with the Parkes radiotelescope. The following large-scale features are distinguished on the $(l, b)$ integrated HI intensity maps: LMC, SMC, Magellanic Bridge joining the two clouds, Magellanic Stream starting at the SMC and following an almost polar plane passing less than about $10^{\circ}$ of the galactic south pole and stretching more than $100^{\circ}$, the Leading Arms - the HI gas preceding the motion of the clouds, and the Interface Region - the HI between Magellanic Bridge and Magellanic Stream (Fig. 2). All these features are also distinguished on the average radial velocity maps. The radial velocity changes smoothly from $R V_{L S R}=-400 \mathrm{~km} \mathrm{~s}^{-1}$ at the end of the Magellanic Bridge to $+100 \mathrm{~km} \mathrm{~s}^{-1}$ at the SMC, to $+240 \mathrm{~km} \mathrm{~s}^{-1}$ at the LMC, while the Leading Arm does not show a clear gradient. Deprojection correcting for the solar motion reduces the velocity difference between the LMC and SMC from $\Delta R V_{L S R}=123 \mathrm{~km} \mathrm{~s}^{-1}$ to $\Delta R V_{G S R}=67 \mathrm{~km} \mathrm{~s}^{-1}$. A further correction taking into account motions of the LMC relative to the MW reduces the difference to $\Delta R V_{L M C S R}=10 \mathrm{~km} \mathrm{~s}^{-1}$. The HI average radial velocities show that the encounter between LMC and SMC happens at a small velocity not much larger that $10 \mathrm{~km} \mathrm{~s}^{-1}$, which makes the interaction rather long, giving the time to the gas to flow away from its parent cloud and form the observed features.

The LMC HI disk seems to be compressed at the side opposite to the SMC, and the LMC shows rotation almost perpendicular to SMC - LMC direction. The LMC disk has a diameter of about $7.3 \mathrm{kpc}$ with a rotation 
curve rising rapidly to $55 \mathrm{~km} \mathrm{~s}^{-1}$ in the inner $1.5 \mathrm{kpc}$, more smoothly to a $63 \mathrm{~km} \mathrm{~s}^{-1}$ peak at $2.4 \mathrm{kpc}$ and declining thereafter (Kim et al., 1998). The SMC also shows rotation: SMC disk includes the bar-like feature about $4 \mathrm{kpc}$ in extent, with a velocity gradient of about $100 \mathrm{~km} \mathrm{~s}^{-1}$ (Stanimirović et al. 2004). The rotation curve rises to about $60 \mathrm{~km} \mathrm{~s}^{-1}$ up to the turnover radius of $3 \mathrm{kpc}$. The velocity dispersion is high along the high column density axis of the Magellanic Bridge. LMC, Magellanic Bridge and SMC show similar velocity fields, which can result from the rotation of all the three partners with the same orientation along the axis LMC - SMC. The velocity field is rather broken at the southern part (in galactic coordinates) of the SMC and of the Magellanic Bridge. There, the Magellanic Stream and Interface Region start. At the other end, in front of the LMC, the Leading Arm can be split into three parts. Individual features in the Leading Arm show head-tail structure with the orientation along the direction of possible space motion of the LMC as it is given by Kroupa \& Bastian (1997) and van der Marel (2001).

The HI observation should be complemented with the studies of distribution of planetary nebulae (Dopita et al. 1985), of carbon stars (Kunkel et al. 2000), and of Cepheids (Groenewegen 2000), and with the near-infrared star counts from the Two Micron All Sky Survey (2MASS) and the Deep Near-Infrared Southern Sky Survey (DENIS). van der Marel (2001) shows that LMC disk is not circular at larger radii, it is elongated in the direction of the Galactic centre, suggesting the influence of the tidal forces of the Milky Way. The data should be compared to models of the interaction in attempts not to be in contradiction.

The N-body simulations modeling the gravitational interaction of the SMC with the LMC and Milky Way (Gardiner \& Noguchi, 1996) show that the last two close encounters between the interaction partners, 1.5 and 0.2 Gyr ago, are able to explain many of the observed structures. Magellanic Stream and Leading Arm have been created as a consequence of the former close encounter, 1.5 Gyr ago. The Magellanic Bridge and the Interface Region have been formed later during the last close encounter between LMC and SMC 0.2 Gyr ago. The discovery of the Leading Arm stressed the importance of gravitational tides and questioned the role of ram pressure stripping (Putman et al. 1998). However, the separation between gaseous and stellar features, absence of stars in tidal Magellanic Bridge and Magellanic Stream, filamentary structures in the SMC and head-tail structures in the Leading Arm and in the Magellanic Stream show that also hydrodynamical forces like ram pressure gas stripping have to act there. Most of the gas in Magellanic Bridge and Leading Arm is coming from the SMC. The preencouter SMC, more that 1.5 Gyr ago, must have a gas disk of about $10 \mathrm{kpc}$ in diameter (Stanimirović 2004), which shrunk forming the Magellanic Stream and Leading Arm 1.5 Gyr ago and Magellanic Bridge and Interface Region 0.2 Gyr ago. However, still a substantial amount of angular momentum of the original disk is left corresponding to other simutions of dwarf galaxies (Mayer et al. 2001) demonstrating that the tidal stripping removes the angular momentum rather slowly, at the timescale of 10 Gyr. Consequently, the recent two encounters 


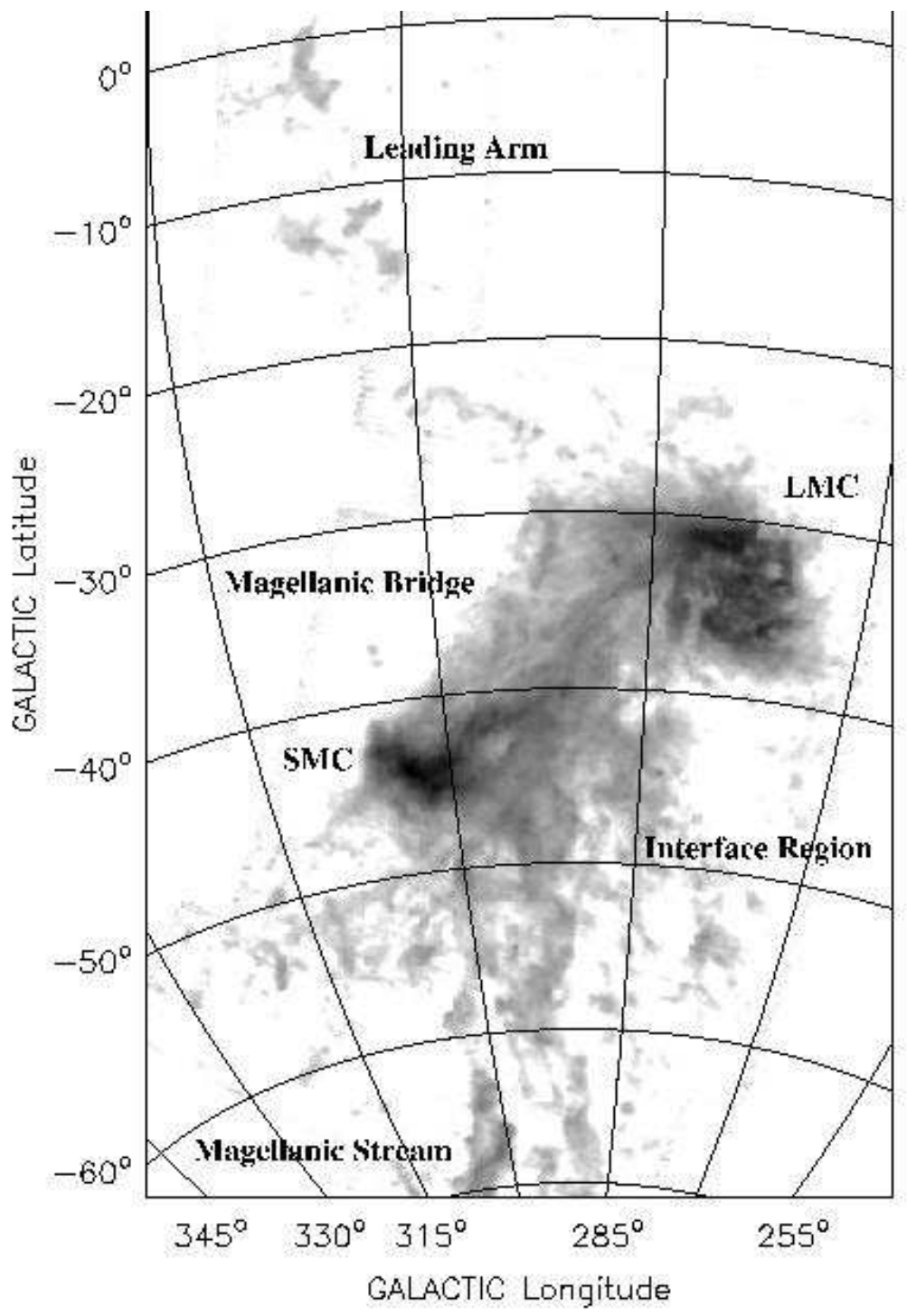

Figure 2: LMC \& SMC: an interacting system of dwarf galaxies (Brüns et al. 2004). 
have not been able to remove a substantial part of the original angular momentum making possible to see the rotation of the SMC at present times. The present rotation curve of the SMC shows that the dynamical mass within 4 kpc is about $2.410^{9} M_{\odot}$ three-quarters of which is stellar. The dark matter is not needed for an explanation of the rotation speeds in the SMC.

Careful analysis of the HI distribution in the Magellanic Stream (Putman et al. 2003) suggests that the Magellanic Bridge is older that assumed above and the LMC and the SMC are bound together for at least two orbits. The dual filaments emanating from the SMC and from the Magellanic Bridge are of tidal origin and shaped by a small amount of ram pressure.

Star formation and energy feedback from young stars are included in the so far most sophisticated N-body model of the LMC - SMC - Milky Way encounter by Yoshizawa \& Noguchi (2003). This model agrees well with several observed features including the Magellanic Stream, which apparently has tidal origin, it reproduces the presence of young stars in the south-east wing of the SMC and it also reproduces the acceleration in the star formation activity, which is due to recent close encounters between the clouds. Some open and unsolved questions remain: bimodal or many peak distribution in the main gaseous body of the SMC and Magellanic Bridge remain to be interpreted, it probably originates in the numerous expanding shells. The shells and supershells, if they trigger star formation, also remain an open question.

The influence of other MW satellites is unclear. The tidal stream of HI clouds connected to disruption of the Sagittarius dwarf galaxy on the polar orbit aloud the Milky Way provides a possible explanation for the anomalous velocity distribution of HI clouds near the south galactic pole (Putman et al. 2003). The possibility of formation of the local group dwarf members including LMC and SMC out of the Milky Way encounter with the M31 galaxy is discussed (Sawa \& Fujimoto, 2004). Another picture describes the interaction of the Fornax-LeoI-LeoII-Sculptor-Sextans stream with the Magellanic Stream causing the gas stripping from the Fornax (Dinescu et al. 2004). Kroupa et al. (2004) propose the origin of the whole Local Group with the local dwarf galaxies in a common great circle.

\section{Star formation, energy and mass feedback}

Star formation is a complex process of the gravitational collapse and fragmentation, where the thermal and magnetic support competes with supersonic shock waves and energy dissipation. The density increases by 20 orders of magnitude from that of a molecular clouds core. The interplay between gravity, magnetic forces, hydrodynamical processes, radiative transfer and chemistry happens in a turbulent interstellar medium. Supersonic flows form sheets and filaments involving mass concentrations, which in some cases are bound by self-gravity. Some of them collapse forming single or binary stars, others disperse.

There are different sources powering the interstellar structures. On a small 
scale the pre-main sequence stellar winds and stellar radiation, on somewhat larger scale the main sequence stellar winds, and on even larger scale the supernovae. Young stars pump energy back to the interstellar medium, which influences the conditions for further star formation. The energy released by young OB associations compresses the ambient medium into shells and supershells, which may collapse and trigger new star formation. The energy feedback triggering shell collapse and further star formation is a self-regulating mechanism of the galaxy evolution.

\subsection{The observation of shells}

Shells and supershells and holes in the HI distribution have been discovered in the Milky Way by Heiles $(1979,1984)$, in M31 by Brinks and Bajaja (1986), in M33 by Deul and Hartog (1990), in LMC by Kim et al. (1999), in SMC by Stanimirovic (1999), in HoII by Puche et at. (1992), in Ho I by Ott et al. (2001) and in IC 2574 by Walter and Brinks (1999). Most probably they are created by an energy release from massive stars, however, an alternative explanations, infall of high velocity clouds (Tenorio-Tagle and Bodenheimer, 1988), or gamma ray bursts (Efremov et al., 1998; Loeb and Perma, 1998) has been invoked in some cases. The majority of the observed shells is due to star formation (Ehlerová and Palouš, 1996). In a new search by Ehlerová and Palouš (2004), more than 600 shells have been identified in the LeidenDwingeloo HI survey of the Milky Way. In Fig. 3 we show the re-identification of a shell previously discovered by Heiles (1979) and a newly discovered shell. The distribution of them in the radial galactocentric direction and in the direction perpendicular to the galactic disk is similar to stellar distribution supporting the idea of a connection between massive stars and shells.

\subsection{The collapse of shells}

We discuss the supersonic expansion of shells and sheets from regions of localized deposition of energy and address the question if and when they fragment and collapse due to gravitational instability. The energy input from an OB association creates a blast-wave which propagates into the ambient medium (Ostriker \& McKee, 1988; Bisnovatyi-Kogan \& Silich, 1995). The schematic representation of the situation is shown in Fig. 4. After the initial fast expansion the mass accumulated in the shell cools and collapses to a thin structure, which is approximated as infinitesimally thin layer surrounding the hot medium inside. Neglecting the external pressure and assuming the constant energy input $L$, the self-similar solution for radius $R$, expansion velocity $V$ and column density $\Sigma_{s h}$ is (Castor et al. 1975; Ehlerová \& Palouš, 2002):

$$
\begin{aligned}
R(t) & =53.1 \times\left(\frac{L}{10^{51} \mathrm{erg} \mathrm{Myr}}\right)^{\frac{1}{5}} \times\left(\frac{\mu}{1.3} \frac{n}{\mathrm{~cm}^{-3}}\right)^{-\frac{1}{5}} \times\left(\frac{t}{\mathrm{Myr}}\right)^{\frac{3}{5}} \mathrm{pc} \\
V(t) & =31.2 \times\left(\frac{L}{10^{51} \mathrm{erg} \mathrm{Myr}{ }^{-1}}\right)^{\frac{1}{5}} \times\left(\frac{\mu}{1.3} \frac{n}{\mathrm{~cm}^{-3}}\right)^{-\frac{1}{5}} \times\left(\frac{t}{\mathrm{Myr}}\right)^{-\frac{2}{5}} \mathrm{kms}^{-1}
\end{aligned}
$$



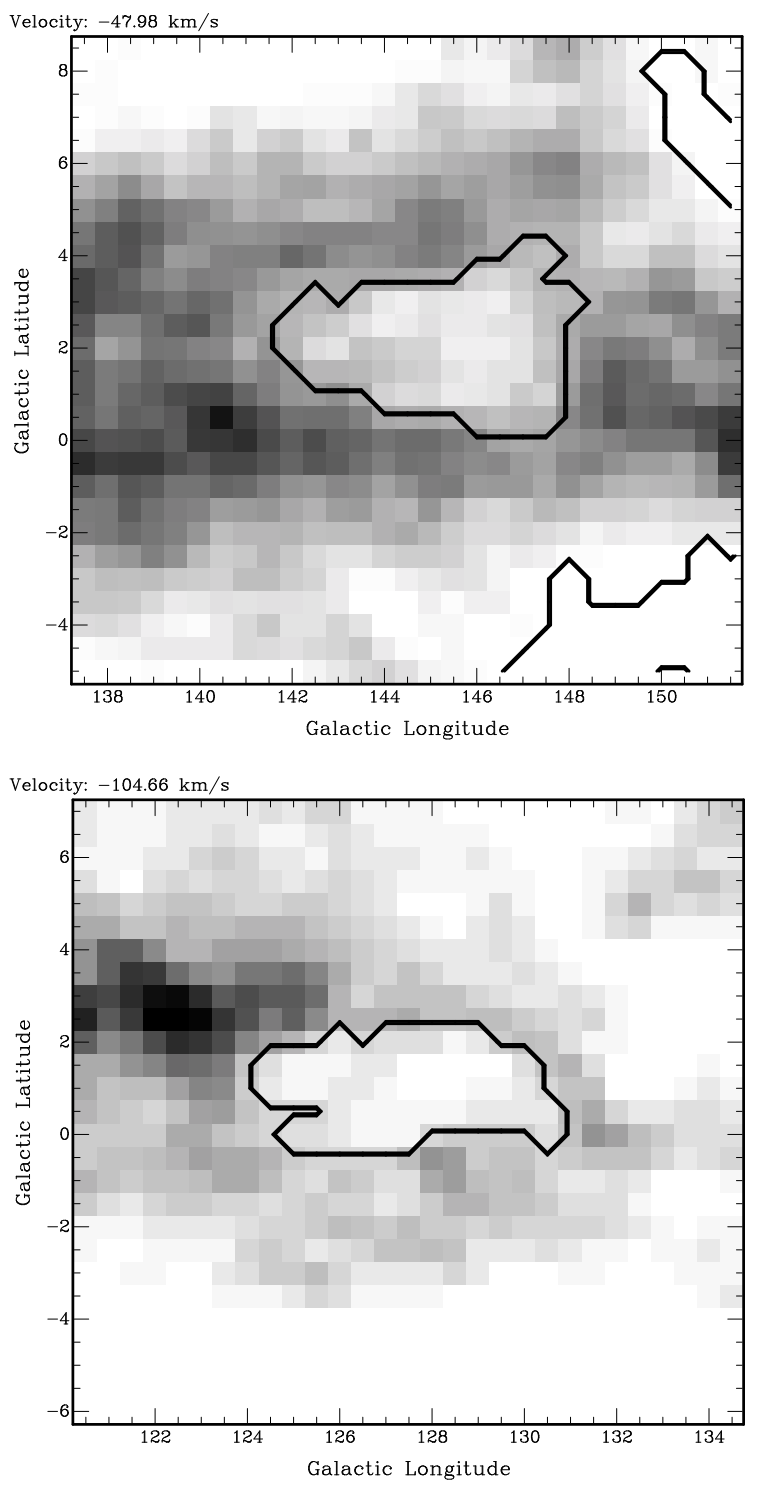

Figure 3: Re-identification of the shell GS 128+01-105 discovered by Heiles (1979) - upper frame, and a newly discovered shell - lower frame. 


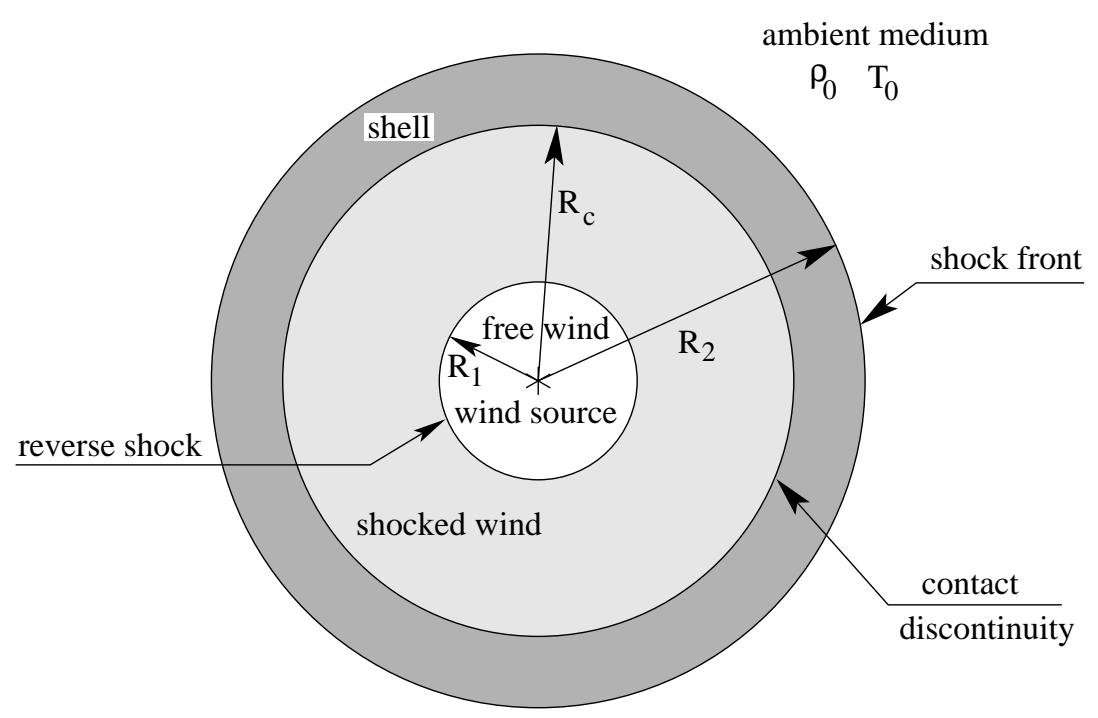

Figure 4: Schematic representation of a supershell expanding around an OB association.

$$
\Sigma(t)_{\mathrm{sh}}=0.564 \times\left(\frac{L}{10^{51} \operatorname{erg~} \mathrm{Myr}^{-1}}\right)^{\frac{1}{5}} \times\left(\frac{\mu}{1.3} \frac{n}{\mathrm{~cm}^{-3}}\right)^{\frac{4}{5}} \times\left(\frac{t}{\mathrm{Myr}}\right)^{\frac{3}{5}} \mathrm{M}_{\odot} \mathrm{pc}^{-2},(6)
$$

where $n$ is the density, $\mu$ is the mean atomic weight of the ambient medium and $t$ is the time since the beginning of an expansion.

The linear analysis of hydrodynamical equations including perturbations on the surface of the shells has been performed by Elmegreen (1994) and Wünsch \& Palouš (2001). The fastest growing mode is:

$$
\omega=-\frac{3 V}{R}+\sqrt{\frac{V^{2}}{R^{2}}+\left(\frac{\pi G \Sigma_{s h}}{c_{s h}}\right)^{2}},
$$

where $c_{s h}$ is the sound speed inside of the expanding shell.

In Fig. 5. we give the time evolution of the fastest mode. At early times, for $t<t_{b}$, the shell is stable. $t_{b}$ is the time, when the fastest mode starts to be unstable:

$$
t_{\mathrm{b}}=28.8 \times\left(\frac{c_{\mathrm{sh}}}{\mathrm{km} \mathrm{s}^{-1}}\right)^{\frac{5}{8}} \times\left(\frac{L}{10^{51} \mathrm{erg} \mathrm{Myr}^{-1}}\right)^{-\frac{1}{8}} \times\left(\frac{\mu}{1.3} \frac{n}{\mathrm{~cm}^{-3}}\right)^{-\frac{1}{2}} \mathrm{Myr} .
$$

Later, for $t>t_{b}$, when the expansion slows down and reduces the stretching, which acts against gravity, and when the shell column density increases, the shell starts to be gravitationally unstable. For ambient densities similar to values in the solar vicinity, $n \sim 10^{-1}-10^{2}$, $t_{b}$ is a few $10^{7} \mathrm{yr}$, which means 


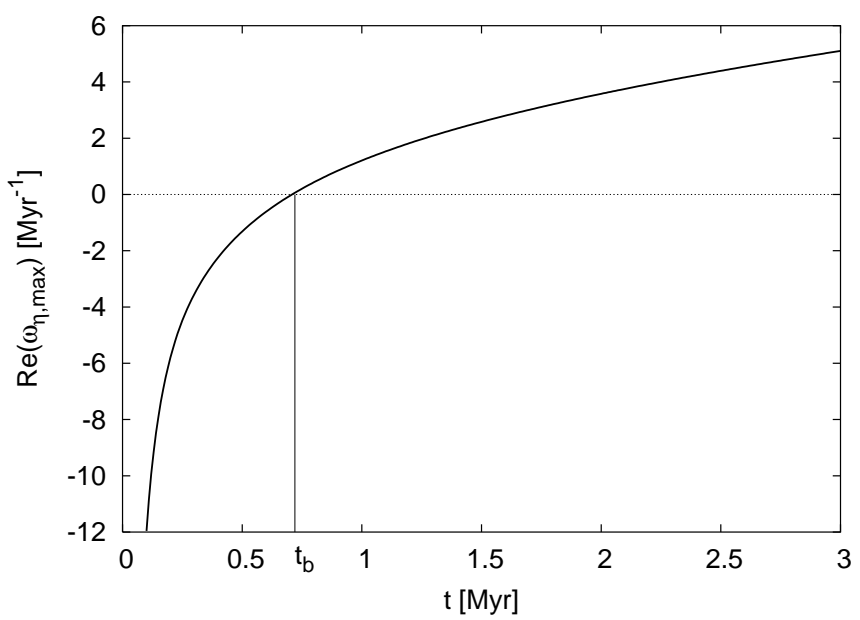

Figure 5: The fastest growing mode.

that the gravitational instability is rather slow compared to turbulent collision times and galactic differential rotation. $t_{b}$ is much smaller in high density medium of GMC and dense cores, where it is $\sim 10^{4}$ yr only. Thus the shell gravitational instability is particularly important inside the GMCs.

The dispersion relation of the shell gravitational instability is:

$$
\omega(\eta, t)=-\frac{3 V}{R}+\sqrt{\frac{V^{2}}{R^{2}}-\frac{c_{s h}^{2} \eta^{2}}{R^{2}}+\frac{2 \pi G \Sigma_{s h} \eta}{R}},
$$

where $\eta$ is the dimensionless wavenumber and $\lambda$ is the wavelength of the perturbation: $\eta=2 \pi R / \lambda$. It is shown in Fig. [6 it gives the wavelength interval of unstable perturbations.

The resulting number of fragments is inversely proportional to the fragment growth time $t_{\text {growth }}=\frac{2 \pi}{\omega(\eta, t)}$. Rapidly growing fragments are more frequent in the final mass spectrum than the slowly growing fragments.

Thus the number of fragments in a given volume of radius $R$ is

$$
N=\omega \frac{R^{3}}{(\lambda / 4)^{3}} .
$$

A fragment with the wavelength $\lambda$ has the mass

$$
m=\frac{4}{3} \pi(\lambda / 4)^{3} \rho .
$$

We derive the mass spectrum $\xi(m)=\frac{d N}{d m}$ :

$$
\xi(m)=-\frac{4}{3} \pi R^{3} \rho \omega m^{-2} .
$$




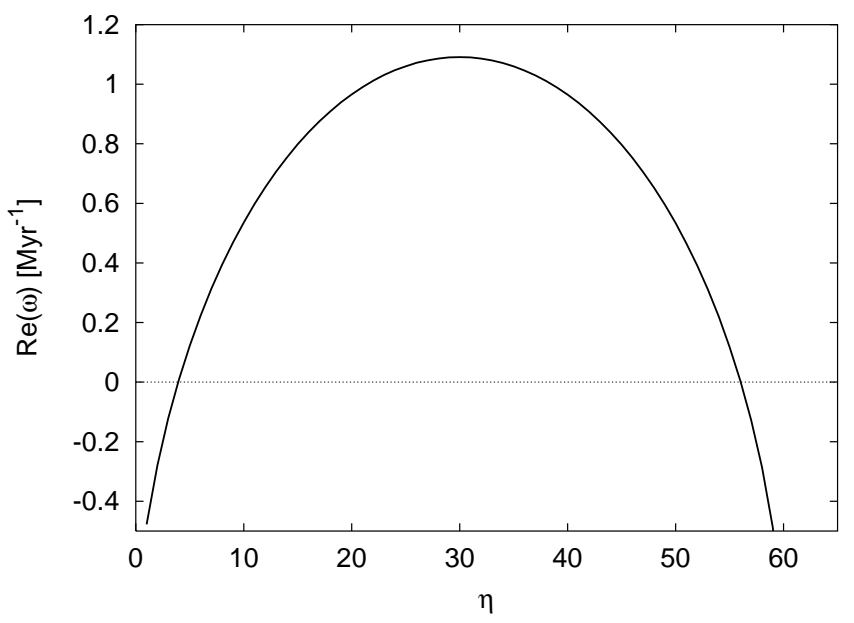

Figure 6: The dispersion relation

If the dispersion relation $\omega(\eta)$ were a constant, the slope of the mass spectrum $\xi(m) \propto m^{-\alpha}$ would be exactly approximated with a power law slope $\alpha=2$. But in our case $\omega$ is not only the function of $\eta$ but also of the time $t$.

We assume that $t_{\text {growth }}$ for a given $\eta$ is inversely proportional to the average value of $\omega$ for this $\eta$ since the time $t_{b}(\eta)$ when a given mode starts to be unstable. The time average $\bar{\omega}$ for a given $\eta$ is calculated using the equation:

$$
\bar{\omega}(\eta)=\frac{\int_{t_{\mathrm{b}}(\eta)}^{t} \omega\left(\eta, t^{\prime}\right) d t^{\prime}}{t-t_{b}(\eta)} .
$$

The resulting mass spectra for different values of $n, c_{s h}$ and $L$, as they have been derived using the thin-shell approximation (4) - (6), are shown in Fig. 7. The high mass parts are well approximated by the power law with a slope $\alpha=2.2-2.4$.

In low density medium, the collapse of expanding shells forms fragments with masses comparable to GMC, however, the collapse time is rather long, a few $10^{7} \mathrm{yr}$. In high density medium of GMC cores, the collapse time is rather short, $\sim 10^{4} \mathrm{yr}$, the masses of individual fragments are close to stellar and the initial mass function of fragments has a power law slope close to the Salpeter (1955) value -2.35. We conclude that the fragmentation of expanding shells qualifies as a possible process triggering the star formation in environments, where the density is high enough, or where it has been increased due to mass accumulation. 


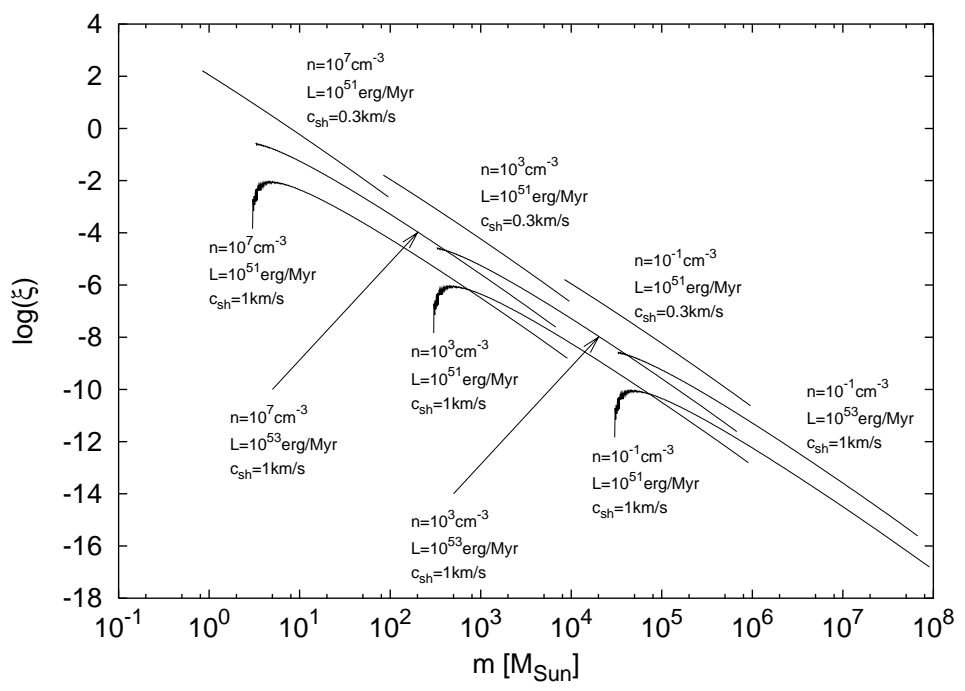

Figure 7: The mass spectrum of fragments of an expanding shell at time $5 t_{\mathrm{b}}$.

\section{Formation of super-star clusters}

Young and massive $\left(10^{5}-10^{7} M_{\odot}\right)$ super-star clusters are observed along tidal arms and bridges of the colliding galaxies. A model of a star forming factory is proposed by Tenorio-Tagle et al. (2003). This model invokes pressure-bounded, self-gravitating, isothermal cloud (Ebert, 1955; Bonner, 1956), which becomes gravitationally unstable when sufficiently compressed. The gravitational instability allows the cloud to enter isothermal collapse. As the collapse proceeds a first generation of stars is formed in the center of the cloud. The mass and energy feedback of the first generation of stars has an important impact on the collapsing cloud. Stellar winds and supernovae compress the infalling material forming a dense shell (see Fig. 8). The shell is able to trap the ionizing radiation and winds of the first generation of young stars. At the same time the shell fragments forming stars with a high efficiency. Thus the cloud, which is compressed in the case of the galaxy encounter, forms a new super-star cluster.

\section{Evolution of the star formation rate}

The investigation of colors in Hubble deep fields (Madau et al., 1996; 1998; Rowan-Robinson, 2003) provides extinction as a function of $z$ : it has been higher at $z=0.5-1.5$ than locally, and lower at $z>2$. Related models of the star formation history show the steep decline of the star formation rate since 


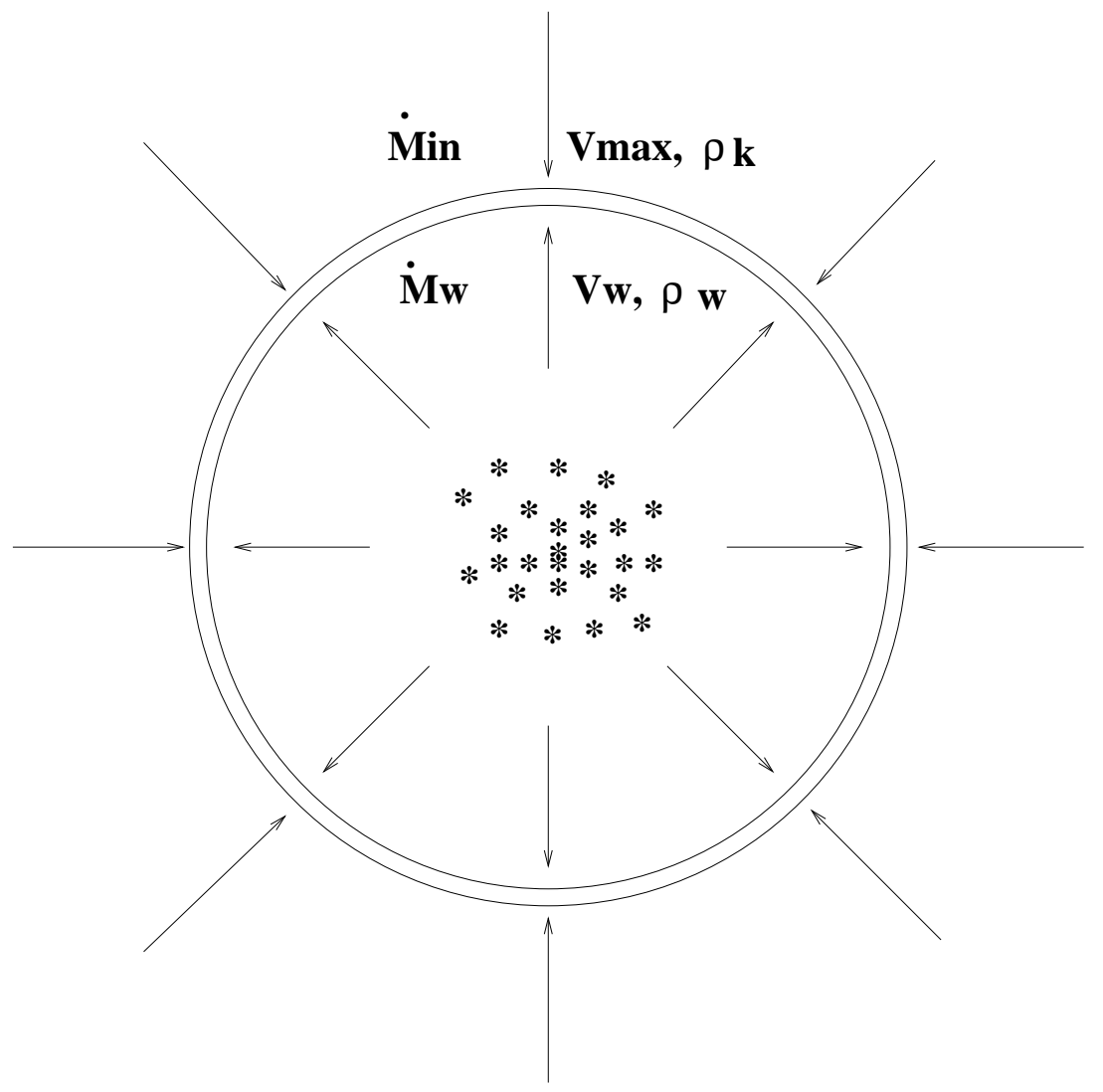

Figure 8: The shell inside of a collapsing cloud 
$z=1$, the present star formation has in average about an order of magnitude smaller rate compared to the level at $z=1-1.5$. Even deeper in the past the correction for the dust extinction remains highly uncertain and a conclusion on the evolution of the star formation rate for $z=2-6$ is difficult.

This opens the question what drives the star formation. Bars in the central parts of galaxies does not seem to change substantially between $z=1$ and present. They do trigger the star formation, however, there has to be more partners in the game. The fuel - the gas - is depleted in galaxies not only due to star formation, but also since the ISM is removed from the galaxy disks with tides, with the ICM ram pressure stripping and with the star formation feedback. Number of galaxy interactions also decreases. We conclude that the decline of the star formation is due to lower rate of triggering from galaxy interactions in a combination with the starvation, since the amount of gas in the star forming disks is reduced by environmental effects and by the star formation itself.

\section{Acknowledgements}

I would like to express my thanks to Guillermo Tenorio-Tagle for comments to the early version of the text.

\section{References}

Abadi, M. G., Moore, B., \& Bower, R. G. 1999, MNRAS, 308, 947

Amram, P., Mercelin, M., Boulsteix, J., \& Le Coarer, E. 1992, A\&A, 266, 106

Amram, P., Mendes de Oliveira, C., Plana, H., Balkowski, C., Hernandez, C., Carignan, C., Cypriano, E. S., Sodré, L. Jr., Gach, J. L., \& Boulsteix, J. 2004, ApJ, 612, L5

Bekki, K., \& Chiba, M. 2002, 566, 245

Bertschik, M., \& Burkert, A. 2003, RevMexAA, 17, 144

Bisnovatyi-Kogan, G. S. \& Silich, S. A. 1995, RvMP 67, 661

Bonner, W. B. 1956, MNRAS, 116, 351

Bournaud, F., \& Combes, F. 2004, A\&A, submitted

Bournaud, F., Combes, F., \& Jog, J. 2004, A\&A, 418, L27

Bournaud, F., Duc, P.-A., Amram, P., Combes, F., \& Gach, J.-L. 2004, A\&A, 425, 813

Brinks, E., \& Bajaja, E. 1986, A\&A, 169, 14

Brüns, C., Kerp, J., Staveley-Smith, L., Mebold, U., Haynes, R. F., Kalberla, P. M. W., Muller, E., \& Filipovic 2004, A\&A, submitted

Bureau, M., \& Carignan, C. 2002, AJ, 123, 1316

Burkert, A. \& Naab, T. 2003a, in Coevolution of Black Holes and Galaxies, ed. L. C. Ho, Carnegie Obs. 
Burkert, A. \& Naab, T. 2003b, in Galaxies and Chaos, ed. G. Contopoulos \& N. Voglis, Springer

Buson, L. M., Bertola, F., Bressan, A., Burstein, D., \& Cappellari, M. 2004, A\&A, 423, 965

Butcher, H., \& Oemler, A. 1978, ApJ, 219, 18

Butcher, H., \& Oemler, A. 1984, ApJ, 285, 426

Castor, J., McCray, R., \& Weaver, R. 1975, ApJ, 200, L107

Chitre, A., \& Jog, C. J. 2002, A\&A, 388, 407

Ciotti, L., Pellegrini, S., Renzini, A., \& D'Ercole, A. 1991, ApJ, 376, 380

Combes, F. 2004, IAU Symp. 217, 440

Couch, W. J., Barger, A. J., Smail, I., Ellis, R. S., \& Sharples, R. M. 1998, ApJ, 497, 188

Deul, E. R., \& den Hartog, R. H. 1990, A\&A, 229, 362

Dinescu, D. I., Keeney, B. A., Majewski, S. R, \& Terrence, G. M. 2004, AJ, 128, 687

Dopita, M. A., Ford, H. C., Lawrence, C. J., \& Webster, B. L. 1985, ApJ, 296, 390

Dressler, A., Oemler, A., Couch, W. J., et al. 1997, ApJ, 490, 577

Duc, P.-A., Bournaud, F.,\& Masset, F. 2004, A\&A, 427, 803

Duc, P.-A., Brinks, E., Springel, V., et al. 2000, AJ, 10, 1238

Ebert, R. 1955, Z. Astrophys., 36, 222

Efremov, Yu. N., Elmegreen, B. G., \& Hodge, P. W. 1998, ApJ, 501, L163

Ehlerová, S., \& Palouš, J. 1996, A\&A, 313, 478

Ehlerová, S., \& Palouš, J. 2002, MNRAS, 330, 1022

Ehlerová, S., \& Palouš, J. 2004, A\&A, submitted

Elmegreen, B. G. 1994, ApJ, 427, 384

Gao, L., Loeb, A., Peebles, P. J. E., White, S. D. M., \& Jenkins, A. 2004, ApJ, 614, 17

Gardiner, L. T., \& Noguchi, M. 1996, MNRAS, 278, 191

Gnedin, O. Y. 2003, ApJ, 582, 141

Groenewegen, M. A. T. 2000, A\&A, 363, 901

Gunn, J. E., \& Gott III, J. R. 1972, ApJ, 176, 1

Heckman, T. M. 2003, RMxAC, 17, 47

Heiles, C. 1979, ApJ, 229, 533

Heiles, C. 1983, ApJS, 55, 585

Hibbard, J. E., van der Hulst, J. M., Barnes, J. M., \& Rich, R. M. 2001, AJ, 122, 2969

Jáchym, P. 2004, in preparation

Jungwiert, B., Combes, F., \& Palouš, J. 2001, A\&A, 376, 85 
Jungwiert, B., Combes, F., \& Palouš, J. 2004, in preparation

Keel, W., Owen, F., Ledlow, M., \& Wang, D. 2004, AAS, 203, 4705

Kenney, J. D. P., \& Koopmann, R. A. 1999, AJ, 117, 181

Kim, S., Staveley-Smith, L., Dopita, M. A., Freeman, K. C., Sault, R. J., Kesteven, M. J., \& McConnell, D. 1998, ApJ, 503, 674

Kim, S., Dopita, M. A., Staveley-Smith, L., \& Bessell, M. S. 1999, AJ, 118, 2797

Koopmann, R. A., \& Kenney, J. D. P. 2004, ApJ, 613, 851

Koopmann, R. A., \& Kenney, J. D. P. 2004, ApJ, 613, 866

Kroupa, P., \& Bastian, U. 1997, New A, 2, 77

Kroupa, P., Theis, Ch., \& Boily, C. M. 2004, A\&A, submitted

Kunkel, W. E., Demers, S., \& Irwin, M. J. 2000, AJ, 119, 2789

Li, Y., Mac Low, M.-M., \& Klessen, R. S. 2004, ApJ, L29

Loeb, A., \& Perma, R. 1998, ApJ, 503, L35

Madau, P., Ferguson, H. C., Dickinson, M. E., Giavalisco, M., Steidel, Ch. C., \& Fruchter, A. 1996, MNRAS, 283, 1388

Madau, P., Pozzetti, L., \& Dickinson, M. 1998, ApJ, 498, 106

Mayer, L., Governato, F., Colpi, M., Moore, B., Quinn, T., Wadsley, J., Stadel, J., \& Lake, G. 2001, ApJ, 559, 754

Mihos, J.Ch. 2004, IAU Symp. 217, eds P.-A. Duc, J. Braine and E. Brinks, p. 390

Mirabel, I. F., Dottori, H., \& Lutz, D. 1992, A\&A, 256, L19

Moore, B. 2003, in Clusters of Galaxies: Probes of Cosmological Structure and Galaxy Evolution, ed. J. S. Mulchaey, A. Dressler, and A. Oemler, CUP

Moore, B., Katz, N., \& Lake, G. 1996, ApJ, 457, 455

Moore, B., Katz, N., Lake, G., Dressler, A., \& Oemler, A. Jr. 1995, Nature, 379, 613

Muller, E. Staveley-Smith, L., Zealey, W., \& Stanimirović, S. 2003, MNRAS, 339, 105

Naab, T., \& Burkert, A. 2003, ApJ, 597, 893

Nulsen, P. 1982, MNRAS, 198, 1007

Ostriker, J. P., \& McKee, C. F. 1988, RvMP 60, 1

Ostriker, J., \& Peebles, J. A. 1973, ApJ, 186, 467

Ott, J., Walter, F., Brinks, E., van Dyk, S. D., Klein, U. 2001, AJ, 122, 3070

Pettini, M., Rix, S. A., Steidel, Ch. C., Shapley, A. E., \& Adelberger, K. L. 2003, IAU Symp. 212, 671

Puche, D., Westpfhal, D., Brinks, E., \& Roy, J. 1992, AJ, 103, 1841

Putman, M. E., Staveley-Smith, L., Freeman, K. C., Gibson, B. K., \& Barnes, D. G. 2003, ApJ, 586, 170

Putman, M. E., Gibson, B. K., Staveley-Smith, L. + 23 co-authors 1998, Nature, 394,752 
Renzini, A. 1997, ApJ, 488, 35

Rezini, A. 2004, in Clusters of Galaxies: Probes of Cosmological Structure and Galaxy Evolution, Cambridge Univ. Press, eds J. S. Mulchaey, A. Dressler, \& A. Oemler, p. 261

Rowan-Robinson, M. 2003, MNRAS, 345, 819

Salpeter, E. E., 1955, ApJ, 121, 161

Sawa, T., \& Fujimoto, M. 2004, astro-ph/0404547

Shen, J., \& Selwood,J. 2004, ApJ, 604, 614

Stanimirović, S., Staveley-Smith, L., Dickey, J. M., Sault, R. J., \& Snowden, S. L. 1999, MNRAS 302, 417

Stanimirović, S., Staveley-Smith, L., \& Jones, P. A. 2004, ApJ, 604, 176

Staveley-Smith, L., Sault, R. J., Hatzidimitriou, D., Kesteven, M. J., \& McConnell, D. 1997, MNRAS, 289, 225

Tenorio-Tagle, G., \& Bodenheimer, P. 1988, ARA\&A, 26, 145

Tenorio-Tagle, G., Palouš, J., Silich, S., Medina-Tanco, G. A., \& Muñoz-Tuñon 2003, A\&A, 411, 397

Toomre, A. 1964, ApJ, 139, 1217

Toomre, A. 1977, ARA\&A, 15, 437

Toomre, A. \& Toomre, J. 1972, ApJ, 178, 623

van der Marel, R. P. 2001, AJ, 122, 1827

van Zee, L., Skillman, E. D., \& Haynes, M. P. 2004, AJ 128, 121

van Gorkom, J. H. 2004, in Clusters of Galaxies: Probes of Cosmological Structure and Galaxy Evolution, Cambridge University Press, Carnegie Observatories Astrophysics Series, eds. J. S. Mulchaey, A. Dressler, and A. Oemler, p. 306

Vollmer, B., Cayatte, V., Balkowski, C., \& Duschl, W. 2001, ApJ, 561, 708

Walter, F., \& Brinks, E. 1999, AJ, 118, 273

Wünsch, R., \& Palouš, J. 2001, A\&A, 374, 746

Yoshizawa, A., \& Noguchi, M. 2003, MNRAS 339, 1135 